\title{
Bioinformatic analysis of fruit-specific expressed sequence tag libraries of Diospyros kaki Thunb.: view at the transcriptome at different developmental stages
}

\author{
Gaurav Sablok • Chun Luo • Wan Sin Lee • \\ Farzana Rahman - Tatiana V. Tatarinova • \\ Jennifer Ann Harikrishna • Zhengrong Luo
}

Received: 30 January 2011 / Accepted: 21 March 2011/Published online: 21 April 2011

(c) The Author(s) 2011. This article is published with open access at Springerlink.com

\begin{abstract}
We present here a systematic analysis of the Diospyros kaki expressed sequence tags (ESTs) generated from development stage-specific libraries. A total of 2,529 putative tentative unigenes were identified in the MF library whereas the OYF library displayed 3,775 tentative unigenes. Among the two cDNA libraries, 325 EST-Simple sequence repeats (SSRs) in 296 putative unigenes were detected in the MF library showing an occurrence of $11.7 \%$ with a frequency of $1 \mathrm{SSR} / 3.16 \mathrm{~kb}$ whereas the OYF library had an EST-SSRs occurrence of $10.8 \%$ with 407 EST-SSRs in the 352 putative unigenes with a frequency of $1 \mathrm{SSR} / 2.92 \mathrm{~kb}$. We observed a higher frequency of SNPs and indels in the OYF library (20.94 SNPs/indels per 100 bp) in comparison to MF library showed a relatively lower
\end{abstract}

G. Sablok and C. Luo are contributed equally to this work.

Electronic supplementary material The online version of this article (doi:10.1007/s13205-011-0005-9) contains supplementary material, which is available to authorized users.

G. Sablok $(\square) \cdot$ C. Luo $\cdot$ Z. Luo $(\square)$

Key Laboratory of Horticultural Plant Biology (MOE),

Huazhong Agricultural University, Shizishan,

Wuhan 430070, China

e-mail: sablokg@gmail.com

Z. Luo

e-mail: luozhr@mail.hzau.edu.cn

W. S. Lee - J. A. Harikrishna

Centre for Research in Biotechnology for Agriculture (CEBAR)

and Genetics and Molecular Biology, Institute of Biological

Sciences, Faculty of Science, University of Malaya,

50603 Kuala Lumpur, Malaysia

F. Rahman · T. V. Tatarinova

Division of Mathematics and Statistics,

University of Glamorgan, Pontypridd CF37 1DL, UK frequency (0.74 SNPs/indels per $100 \mathrm{bp}$ ). A combined homology and secondary structure analysis approach identified a potential miRNA precursor, an ortholog of miR159, and potential miR159 targets, in the developmentspecific ESTs of D. kaki.

Keywords Diospyros kaki . Expressed sequence tag · $\mathrm{GC}_{3}$ biology $\cdot$ MicroRNA $\cdot$ SSRs $\cdot$ SSR-FDM $\cdot$ SNPs

\begin{tabular}{|c|c|}
\hline \multicolumn{2}{|c|}{ Abbreviations } \\
\hline AFLP & Amplified fragment length polymorphism \\
\hline IRAP & $\begin{array}{l}\text { Inter-retrotransposon amplified } \\
\text { polymorphism }\end{array}$ \\
\hline MF library & Mature fruit library \\
\hline ORF & Open reading frame \\
\hline OYF library & Ovary and young fruit library \\
\hline RAPD & Random amplified polymorphic DNA \\
\hline RFLP & Restriction fragment length polymorphism \\
\hline REMAP & $\begin{array}{l}\text { Retrotransposon microsatellite amplified } \\
\text { polymorphism }\end{array}$ \\
\hline SRAP & Sequence-related amplified polymorphism \\
\hline SNPs & Single nucleotide polymorphisms \\
\hline SSAP & Sequence-specific amplified polymorphic \\
\hline SSRs & Simple sequence repeats \\
\hline SSR-FDMs & $\begin{array}{l}\text { Simple sequence repeats-functional domain } \\
\text { markers }\end{array}$ \\
\hline
\end{tabular}

\section{Introduction}

The genus Diospyros (Ebenaceae) is a widely distributed heterozygous genus in tropic and subtropic areas of Asia, Africa, and America (Central and North) with complex ploidy levels ranging from diploid $(2 n=2 x=30)$ to 
nanoploid $(2 n=9 x=135)$ (Yonemori et al. 2000). Diospyros kaki Thunb. (classified by a prominent Swedish naturalist Carl Peter Thunberg) or Japanese persimmon is the most economically important climacteric fruit species (having varied levels of ploidy; $2 n, 6 n$ and $9 n$ ) within this genus. In 2007 , gross production of persimmon was estimated to be about 2,340,000 tons, of which $89.8 \%$ was produced in China, one of the origins of Japanese persimmon (Wang et al. 1997; FAO 2008). Vitamins A and C constitute the major portion of the vitamins present in fresh persimmon fruit.

On the basis of proanthocyanidins (PAs) (colorless phenolic polymers known as useful agents for human health, which show brown coloration upon oxidation), persimmon are further classified into astringent (A)-type fruits and nonastringent (NA)-type fruits (Dixon 2005; Ikegami et al. 2009). A comparative analysis of catechin composition among the five Japanese persimmon demonstrated that epigallocatechin (EGC) is relatively lower in the nonastringent type persimmon (Suzuki et al. 2005). An AST/ast allele having allelotypes as astringent (A) and non-astringent (NA) controls the expression of the trait. Expression of homozygous recessive ast allelotype at the AST locus results in the non-astringent (NA) genotype (Kanzaki et al. 2001; Yamada and Sato 2002). Recently, Ikegami and her team (2007) isolated seven genes (PAL, C4H, CHI, F3H, F3OH, $A N S$, and $A N R$ ) from an astringent-type cultivar using suppression subtractive hybridization. Transcription of $P A L$, C4H, 4CL, CHS, CHI, F3H, F3OH, F305OH, DFR, and ANR genes is high until mid-August, and then declines in October in the astringent-type cultivar (Ikegami et al. 2005a, b). Pang et al. (2007) identified ethylene receptor genes (DkERS1, DkETR1, and DkETR2) homologous to Arabidopsis ethylene receptor genes (ERS1, ETR1, and ETR2) in D. kaki. A Myb transcription factor (DkMyb4) controls proanthocyanidin biosynthesis in persimmon fruit (Akagi et al. 2009). Overall, D. kaki has great potential for becoming a model for understanding important traits in tannins and flavonoid biosynthesis as a fruiting crop species.

Diospyros kaki fruiting genotypes have wide morphological, physiological, and molecular diversity. Dominant and transposon-based markers have been described for Diospyros, including RAPD, RFLP, IRAP, REMAP, SSAP, SRAP, and AFLP (Yamagishi et al. 2005; Guo and Luo 2006; Du et al. 2009a, b).

In recent years, using in silico approach through mining expressed sequence tags (ESTs) have become an effective way for developing molecular markers such as Simple sequence repeats (SSRs), SNPs, SSR-FDMs for developing the saturated genetic linkage maps for various plant species (Hytena et al. 2010). In addition, the markers so developed not only exhibit higher level of intragenic transferability but also transferability to other closely related genera and may serve as potential markers for species discrimination, evolutionary inference and comparative genomics (Varshney et al. 2005). Extensive analysis has been done using ESTs available in the publicly available databases to identify genes temporally or spatially regulated during fruit growth and development in tomato, grape and apple (Fei et al. 2004; Da Silva et al. 2005; Park et al. 2006).

One of the most recently discovered regulatory mechanisms is post-transcriptional and involves 21-24-nt small RNA molecules (sRNAs). Micro RNAs (miRNAs) are nonprotein coding, genomic derived small RNAs that participate in regulation of gene expression at a post-transcriptional level. In plants, they are involved in development, responses to biotic and abiotic stress and whilst some appear unique to a species, a large number of miRNA families are highly conserved across a wide range of plant species (Yang et al. 2007; Jian et al. 2010). miRNA transcripts are capped, spliced, polyadenylated and folded into long hairpin stem-loop precursor molecules (pre-miRNA), which are then processed by RNase III enzymes (Dicer-like in plants; $D C L 1$ ) to form shorter hairpin primary miRNAs (Zhang et al. 2006). A 21-23 base pair double-stranded miRNA: miRNA* duplex is produced by further action of the Dicer enzyme and transported to the cytoplasm where the single-stranded mature miRNA is used as a template for target mRNAs silencing with complementary sequences by cleavage or translational inhibition by an RNA-induced silencing complex (RISC) (Bartel 2004; Zhang et al. 2006). The high sequence conservation of mature plant miRNAs has led to their successful prediction from sequence data using homology-based approaches (Zhang et al. 2005; Sunkar and Jagadeeswaran 2008).

We report here comparative mining of fruit cDNA libraries from different developmental stages of $D$. kaki. We have identified 506 SSRs primer pairs that can be further utilized for the inference of genetic diversity, species discrimination and studying the phylogeography of Diospyros genus and in particular D. kaki, a potential ortholog of miRNA159 in OYF library, which correlates the potential involvement of miR 159 family in development and relative distribution of SNP and SSR-FDMs markers.

\section{Materials and methods}

Sequence source and assembly

Diospyros kaki ESTs sequences were downloaded from GenBank (dbEST http://www.ncbi.nlm.nih.gov/dbEST) to give a total of 5,053 D. kaki ESTs from OYF library and 4,404 D. kaki ESTs from MF library. Mature miRNA sequences for all plant species were retrieved from the miRBase Registry (Release 14, September 2009, http:// microrna.sanger.ac.uk/) and were used to generate a 
non-redundant reference set of 1,064 mature miRNA sequences. EST sequences were clustered using CAP3 program to prepare a tentatively consensus (TC) set (Huang and Madan 1999). To compare the relative richness of gene diversity sampled from each library, library-specific contigs, and singletons were compared.

\section{SSR identification}

The identification of SSR containing ESTs was carried out using in-house written program in $\mathrm{C}$, which gives perfect as well as compound SSRs. Repeat patterns ranging from mono- to hexa-nucleotide were identified and systematically analyzed. The parameters defined for the identification of simple sequence repeats were seven minimal repeats for di-, five minimal repeats for tri-, four minimal repeats for tetra- and penta-, and three minimal repeats for hexa-nucleotide. The minimal length of mononucleotide simple sequence repeat was fixed at $14 \mathrm{bp}$. The poly A and poly $\mathrm{T}$ repeats were not considered as SSRs as they exemplify the $3^{\prime}$ end of mRNA/cDNA sequences, thus they were removed. Compound microsatellites were defined as repeats interrupted by a non-repetitive stretch of a maximum of 100 nucleotides.

\section{SNP identification}

Expressed sequence tags sequences were trimmed and a redundancy-based method for SNP confidence measurement, combined with SNP co-segregation (an independent confidence measure) was used to mine SNPs (Barker et al. 2003). The co-segregation score is a measure of whether a predicted SNP contributes to the definition of haplotype. The transition $\left(T_{\mathrm{s}}\right)$ versus transversion $\left(T_{\mathrm{v}}\right)$ ratio was also calculated for both the libraries to find the DNA substitution dynamics in the D. kaki genome.

Locus-specific primer designing and prediction of SSRs in open reading frames to identify relative biasing

Primer 3 software was being used to design a pair of primers flanking each SSR. The following parameter were used while designing the SSRs primers-optimum primer size was set to 20 where the range was between 18 and 27 , optimum annealing temperature was set to 60.0 (the range was between 57.0 and 63.0), and the range of GC content was 20-80\% (Shanker et al. 2007). Custom scripts and the standard genetic codes were applied to predict ORFs for all SSR-ESTs. SSR-ESTs were translated in all six ORFs and the longest fragments uninterrupted by stop codons were taken as the putative encoding segment (ORF) of the query SSR-ESTs sequences.
Annotation of SSR containing sequences, $\mathrm{GC}_{3}$ biology and gene ontology

Functional annotations of the SSR-ESTs sequences were determined on the basis of similarity using BLASTX program, available at NCBI (http://www.ncbi.nlm.nih.gov/ blast) against non-redundant (nr) protein database entries and the best matches ( $E$ value $<10^{-10}$ ) were compared to terms of the Gene Ontology (GO) Consortium (The Gene Ontology Consortium 2000). The resulting proteins obtained through similarity search by BLASTX were allotted to their respective classes. Using $\mathrm{GO} / \mathrm{UniProt}$ comparison tables, candidate GO assignments were predicted on the basis of EST matches to the UniProt reference sequences.

Coding sequences of four additional Ericales species, such as: Actinida deliciosa, A. chinesis, Vaccinium corymbosum and Camellia sinensis were obtained from NCBI; in-house $\mathrm{C}++$ code was used to compute position-specific nucleotide composition. In case of $D$. kaki, open reading frames and corresponding proteins were predicted using the assembled contigs of ESTs and nucleotide composition and sequence length was computed for each of the two EST libraries separately.

Using Gene Ontology (The Gene Ontology Consortium 2000) annotation of Arabidopsis thaliana (available at http://www.arabidopsis.org), all D. kaki protein sequences were aligned to $A$. thaliana using NCBI blastp with $E$ value cut-off of $10^{-30}$, and the GO annotation of the best hit was used to annotate $D$. kaki genes. Chi-squared test $(\alpha=0.05)$ was used to identify significant enrichment of different GO categories in high- and low- $\mathrm{GC}_{3}$ genes (Tatarinova et al. 2010). Categories were assigned on the basis of biological, functional, and molecular annotations available from the GO website (http://www.geneontology.org/).

Identification of functional domains markers (SSRFDMs)

Using a python script sequences were translated into all six reading frames. In addition, Inter pro scan tool was used to analyze protein domain maintaining default parameter value (Quevillon et al. 2005; Yu et al. 2010). The sequences that contained both SSRs and functional protein domains were selected as SSR-FDMs; however, absence of predicted protein (as non-functional domain) caused exclusion for the sequences from further analysis.

Homology search and secondary structure prediction for miRNA identification

Candidate miRNA precursor sequences within the EST data were identified using BLAST and MFOLD RNA 
folding algorithms with parameters described elsewhere (Nasaruddin et al. 2007). Briefly, standalone BLAST (ver. BLAST-2.2.16) was used for local alignment of the EST against the non-redundant query set of 1,064 plant mature miRNA sequences. Default settings were as described elsewhere (Zhang et al. 2005). ESTs sharing homology with miRNAs in the reference set were defined as those containing a predicted mature miRNA with less than four $(<4)$ mismatches compared to a known mature miRNA sequence in the reference set. Putative miRNA orthologs were analyzed using MFOLD RNA folding program (http://mfold.bioinfo.rpi.edu/ cgi-bin/rna-form1.cgi) and candidate precursor miRNA (pre-miRNA) were filtered using the characteristics described elsewhere (Zuker 2003; Nasaruddin et al. 2007; Qiu et al. 2007; Xie et al.2007). Briefly, (1) the composition of the RNA sequences needs to be folded into a hairpin structure as per the stem-loop precursors. According to this process, each arm of the hairpin will contain $\sim 22 \mathrm{nt}$ mature miRNA sequences; (2) the lower minimal-free energy (MFE) and minimal-free energy index (MFEI) should be compulsorily present in the predicted secondary structure of the miRNA precursors than the tRNA or rRNA; (3) 30-70\% of A + U content should be present in the predicted mature miRNA; (4) the mature miRNA sequence is the integral part of the hairpin loop segment. This mature miRNA should have less than six mismatches to the opposite miRNA* sequence of the other arm; (5) any part of mature miRNA:RNA*dimer loop or bulge should contain three nucleotides (maximum). This nucleotides should not be involved in canonical base pairing.

\section{Prediction of miRNA targets}

Potential targets of strong candidate miRNA from D. kaki EST were anticipated using RNA hybrid (http://bibiserv.techfak.uni-bielefeld.de/rnahybrid/) (Rehmsmeier et al. 2004). The mature miRNA sequence was used to query the complete EST dataset using the following parameters: helix constraint $(-f)$ of $8-12$; maximum internal loop size $(-u)$ of one and maximum bulge loop size $(-v)$ of one (Rehmsmeier 2006). Good candidates were considered those with a negative folding free energy (MFE; $\Delta \mathrm{Kcal} / \mathrm{mol}$ ) value below $70 \%$ of the MFE value for perfect complementarily and with end overhangs of no more than two nucleotides (Alves et al. 2009). Function of ESTs were predicted using BLASTX program by comparing the sequences against the nonredundant NCBI protein database with a cut off $E$ value of $10^{-4}$ and $40 \%$ minimum identity score.

\section{Results and discussion}

Sequence assembly

Expressed sequence tags, represent partial and redundant cDNA sequences, making it difficult to analyze them effectively for putative mining of markers. To construct longer and less redundant sequence sets, we assembled ESTs by library, using CAP3 (Huang and Madan 1999). In OYF library, clustering of 5,053 sequences yielded 658 tentative consensus (TC) sequences with 3,117 sequences remained unclustered. In MF library, clustering of 4,404 sequences yielded 604 tentative consensus (TC) sequences with 1,925 sequences as singletons. The average length of the tentative consensus (TC) was 521 and 368 bp in OYF and MF library respectively. The diversity in ESTs libraries was confirmed by diversity index depicting higher degree of transcript diversity (Table 1).

Screening, frequencies, primer designing and annotation of $D$. kaki SSRs-ESTs

In the present study, library-specific tentative consensus (TC) set of $D . k a k i$ were mined for SSRs with a minimum length of $14 \mathrm{bp}$. A total of 407 and 325 SSRs were detected in the OYF and MF libraries respectively; excluding poly

Table 1 Summary of in silico mining of Diospyros kaki cDNA libraries for assembly and repeat analysis

\begin{tabular}{lll}
\hline Parameters & \multicolumn{2}{l}{ Values } \\
\cline { 2 - 3 } & OYF Library & MF Library \\
\hline Total number of EST & 5,053 & 4,404 \\
Total number of contigs & 658 & 604 \\
Total Number of ESTs left to assemble & 3,117 & 1,925 \\
Redundancy index (\%) & 38.31 & 56.28 \\
Total number of unigenes sequences & 3,775 & 2,529 \\
searched & & \\
Total number of SSRs after removing & 407 & 325 \\
poly A and poly T & & \\
Average UniGene length including & 521.12 & 367.56 \\
poly A and poly T & & \\
Diversity index (\%) & 75 & 57.4 \\
Repeat type & & \\
Mono-nucleotide & $3(0.73)^{*}$ & $4(1.23)$ \\
Di-nucleotide & $229(56.3)$ & $175(53.84)$ \\
Tri-nucleotide & $101(24.81)$ & $98(30.2)$ \\
Tetra-nucleotide & $18(4.42)$ & $20(6.2)$ \\
Penta-nucleotide & $4(1.0)$ & $6(1.8)$ \\
Hexa-nucleotide & $52(12.8)$ & $22(6.8)$ \\
\hline
\end{tabular}

* Data in parentheses is the percentage value of the repeat type 
A and poly T. Poly (A/T) were excluded (Karaoglu et al. 2004). In the OYF library, 5,053 sequences represent 407 SSRs with an average density of one SSR per $2.92 \mathrm{~kb}$ whereas in MF library from a number of 4,404 sequences screened only 325 SSRs were detected demonstrating average density of one SSR per $3.16 \mathrm{~kb}$. The frequencies of SSRs with mono-, di-, tri-, tetra-, penta- and hexanucleotide repeat units are shown in Table 1 . The most frequent repeat type found among different developmental libraries analyzed were di-nucleotide repeats $(53.8 \% ; 53.6 \%)$ followed by tri-nucleotide $(30.2 \% ; 24.8 \%)$, hexa-nucleotide $(6.8 \% ; 12.8 \%)$, tetra-nucleotide $(6.2 \% ; 4.4 \%)$, pentanucleotide $(1.8 \% ; 1.0 \%)$, and mono-nucleotide repeats $(1.2 \% ; 0.7 \%)$, respectively (Fig. 1$)$.

In both cDNA libraries surveyed, the mono-nucleotide repeats were relatively low when compared with other repeats. We further analyzed the observed abundant dinucleotide and trinucleotide repeat patterns (Figs. 2, 3) and reduction in the frequency of SSRs before and after assembly is both the libraries (Table 2). Similar patterns have been observed in the mining of the EST-SSRs markers in cereal species (Varshney et al. 2002). In case of the OYF library, out of 407 SSRs detected, primers could be designed only for $286(70.2 \%)$ SSRs, whereas for the MF library, out of 325 SSRs detected, primers could be designed only for $220(67.6 \%)$ SSRs (Supplementary

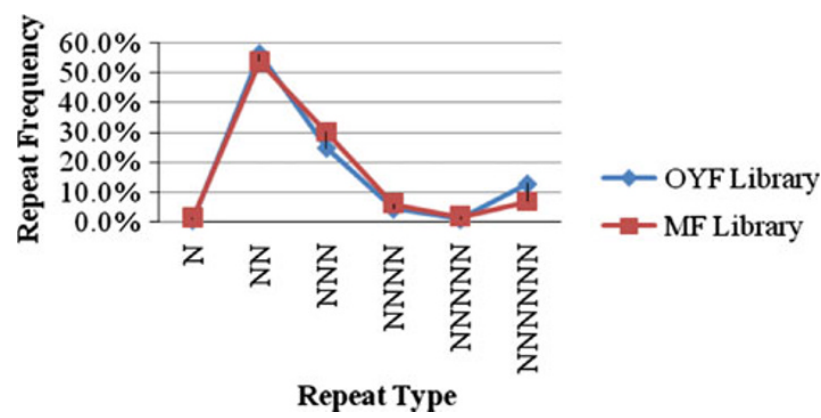

Fig. 1 Frequency distribution of library specific repeat types identified in Diospyros kaki

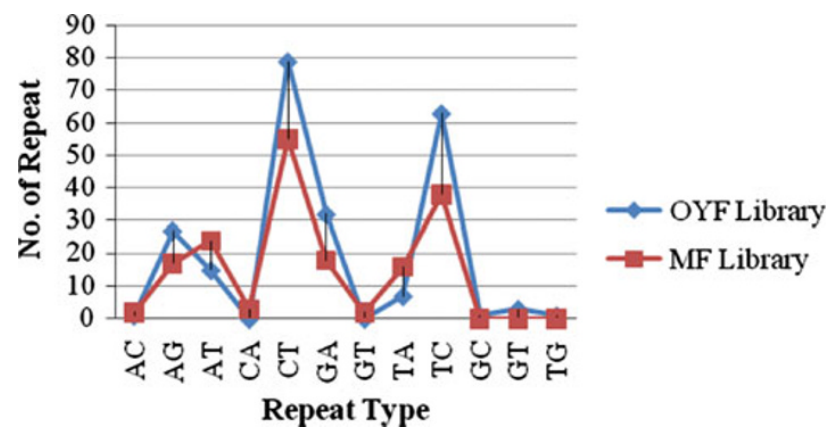

Fig. 2 Frequency distribution of library specific dinucleotide repeat types identified in Diospyros kaki
Table 1). SSRs with primer pairs with respect to ORF were predicted in both MF and OYF libraries. In OYF library, out of 407 SSRs identified, 220 SSRs with primer pairs $(54.0 \%)$ were found with respect to ORF. In the MF library, out of 325 SSRs identified, 153 (47.0\%) SSRs with primer pairs were found with respect to ORF (Fig. 4).

Genic as well as the intergenic regions displayed the presence and absence of SSRs (Katti et al. 2001). A higher percentage of dinucleotide repeats in non-ORF regions may reflect natural evolution to maintain the conservation of functionality of all genes and their products (Fig. 4). Nevertheless, recent studies indicate that SSR expansions and/or contractions in protein-coding regions may cause a gain or loss of gene function through frame shift mutations (Fondon et al. 2008).

Simple sequence repeat-containing sequences (SSRsESTs) were annotated against the non-redundant (nr) protein database available at http://www.ncbi.nlm.nih.gov. Out of 296 MF library-derived SSRs-ESTs, 131 were found to homologous (44.3\%) whilst for the OYF library, out of 352 SSRs-ESTs, homologs were available for only 146 $(41.5 \%)$ sequences.

\section{SNP identification}

Redundancy-based SNPs mining resulted in identification of 68,067 SNPs and 4,273 indels in the D. kaki transcriptome. SNPs occurred at a frequency of one out of every $10 \mathrm{bp}$ and indels at one in every $152 \mathrm{bp}$. A total of 28,232 transitions and 39,835 transversions were reported in this study. For explaining the nucleotide substitution dynamics, transition $\left(T_{\mathrm{s}}\right)$ to transversion $\left(T_{\mathrm{v}}\right)$ ratio was calculated because it provides insights into the process of molecular evolution. The transition/transversion ratio is relatively low in the OYF library (0.69) compared to the MF library (1.39) the overall transition $\left(T_{\mathrm{s}}\right)$ to transversion $\left(T_{\mathrm{v}}\right)$ ratio is 0.70 , which indicates an relative increase of transversion $\left(T_{\mathrm{v}}\right)$ over transitions $\left(T_{\mathrm{s}}\right)$ (Table 3$)$.

Earlier studies have demonstrated higher rate of transitions over transversions due to abundant hypermutable methylated dinucleotides (5'-CpG-3') (Ching et al. 2002; Strandberg and Salter 2004; Newcomb et al. 2006). However, in the present study, transversions prevail over the transitions. Neighbouring nucleotide effect demonstrates that the probability of transversion increases when the number of purines increases at the immediate adjacent sites (Zhongming and Boerwinkle 2002). Similar patterns of transversions over transitions were observed for genes on rice chromosome 8 (Wu et al. 2004).

In plant chloroplasts, an increase in transversions with increase in the $\mathrm{A}+\mathrm{T}$ content of adjacent nucleotides has been observed (Morton 1995). These studies illustrate that the transition bias is not universal and supports the findings 
Fig. 3 Frequency distribution of library specific trinucleotide repeat types identified in Diospyros kaki

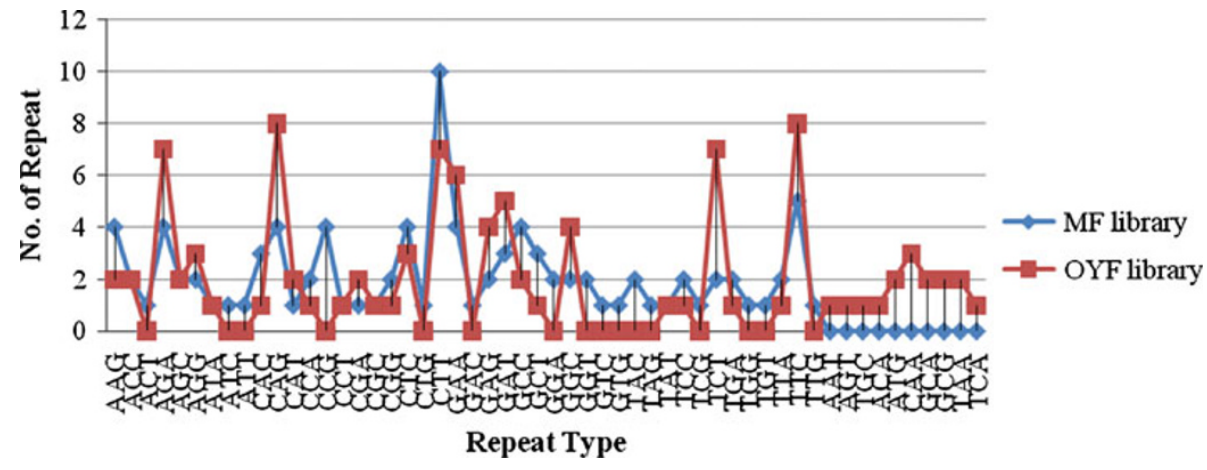

Table 2 Frequency of SSRs in the EST sets before and after assembly

\begin{tabular}{|c|c|c|c|c|c|c|}
\hline \multirow{2}{*}{$\begin{array}{l}\text { Source of } \\
\text { ESTs }\end{array}$} & \multicolumn{3}{|c|}{ Before assembly } & \multicolumn{3}{|c|}{ After assembly } \\
\hline & $\begin{array}{l}\text { No. of } \\
\text { sequence }\end{array}$ & $\begin{array}{l}\text { No. of SSR-ESTs } \\
\text { (1-6 bp) }\end{array}$ & $\begin{array}{l}\text { SSR-ESTs } \\
(\%)\end{array}$ & $\begin{array}{l}\text { No. of } \\
\text { unigenes }\end{array}$ & $\begin{array}{l}\text { No. of SSR-ESTs } \\
(1-6 \mathrm{bp})\end{array}$ & $\begin{array}{l}\text { SSR-ESTs } \\
(\%)\end{array}$ \\
\hline OYF library & 5,053 & 586 & 11.5 & 3,775 & 407 & 10.78 \\
\hline MF library & 4,404 & 611 & 13.8 & 2,529 & 325 & 12.8 \\
\hline
\end{tabular}

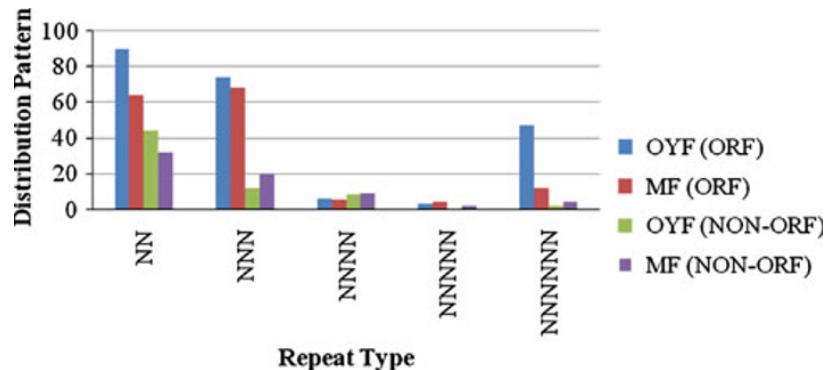

Fig. 4 Relative distribution patterns of SSRs with primer pairs in ORF and Non-ORF among di-, tri-, tetra-, penta- and hexa-nucleotide repeat in OYF and MF library

of the present study. However, the frequency of SNPs detected in this study is higher than the frequency of EST derived SNPs generally reported in earlier studies; $1 \mathrm{SNP} /$ 61 bp in Zea mays, 1 SNP/540 bp in Triticum aestivum, 1 $\mathrm{SNP} / 123$ bp in Sorghum bicolor and1 SNP/58 bp in Secale cereale transcriptome (Ching et al. 2002; Somers et al. 2003; Hamblin et al. 2004; Varshney et al. 2007). Possible reasons for variation in SNP density may perhaps be due to dissimilarity in the quantity of data analyzed.

Functional domains markers (SSR-FDMs)

Tentative consensus (TC) from the respective libraries was analyzed for functional domain markers excluding the mononucleotide repeats from this analysis. The translation of the sequences was performed in all six reading frames. InterProScan tool was used to analyse the resulting amino acid sequences from the longest reading frame (http:// www.ebi.ac.uk/Tools/pfa/iprscan/). In the case of the OYF library, four potential SSR-FDMs were observed and Vps4 oligomerisation domain and the $\mathrm{C} 2$ calcium/lipid-binding domain were identified as major functional domains. The MF library displayed 10 potential SSR-FDMs but Basicleucine zipper (bZIP) transcription factor, Glycoside

Table 3 SNP analysis

\begin{tabular}{lllll}
\hline S. No. & Parameter & OYF Library & MF library & Total \\
\hline 1 & Total sequences analyzed & 1,936 & 2,479 & 4,415 \\
2 & Total number of TC sequences & 658 & 604 & 1,262 \\
3 & Total SNP and indels detected & 70,008 & 2,332 & 72,340 \\
4 & Total consensus size (bp) & 334,271 & 314,897 & 649,168 \\
5 & Total transitions & 27,016 & 1,216 & 28,232 \\
6 & Total transversions & 38,966 & 869 & 39,835 \\
7 & Total indel & 4,026 & 247 & 4,273 \\
8 & $T_{\mathrm{s}} / T_{\mathrm{v}}$ & 0.69 & 1.39 & 0.7 \\
9 & SNP frequency & $1 \mathrm{SNP} / 5 \mathrm{bp}$ & $1 \mathrm{SNP} / 151 \mathrm{bp}$ & $1 \mathrm{SNP} / 10 \mathrm{bp}$ \\
10 & Indel frequency & 1 Indel/83 bp & 1 Indel/1,274 bp & 1 Indel/152 bp \\
\hline
\end{tabular}


Table 4 ESTs from Libraries for ovary and young fruit and for mature fruit less than four mismatches to mature miRNA from rice or from Arabidopsis

a Identifiers from miRBase Registry. Only the highest scoring match is shown

${ }^{b}$ Identifiers for EST from the Mature Fruit library

\begin{tabular}{lllll}
\hline S. No. & miR Family $^{\mathrm{a}}$ & $\begin{array}{l}\text { Length of } \\
\text { mature miRNA }\end{array}$ & $\begin{array}{l}\text { Match } \\
\text { (BLAST) }\end{array}$ & EST \\
\hline 1 & osa-miR414 & 21 & $18 / 21$ & DC588681.1 $^{\mathrm{b}}$ \\
2 & ath-miR414 & 21 & $18 / 18$ & DC591906.1 $^{\mathrm{b}}$ \\
3 & osa-miR395o & 21 & $20 / 21$ & DC591801.1 $^{\mathrm{b}}$ \\
4 & peu-miR2914 & 23 & $22 / 22$ & DC592202.1 \\
5 & ppt-miR1038-3p & 21 & $18 / 20$ & DC589073.1 \\
6 & peu-miR2910 & 21 & $21 / 21$ & DC589557.1 \\
7 & osa-miR159a.1; osa-miR159b & 21 & $21 / 21$ & DC584412.1 \\
8 & sof-miR408e & 21 & $19 / 20$ & DC585074.1 \\
9 & osa-miR408 & 21 & $18 / 18$ & DC584676.1 \\
10 & osa-miR414 & 21 & $18 / 20$ & DC588395.1 \\
11 & ath-miR414 & 21 & $19 / 20$ & DC588139.1; DC586156.1 \\
\hline
\end{tabular}

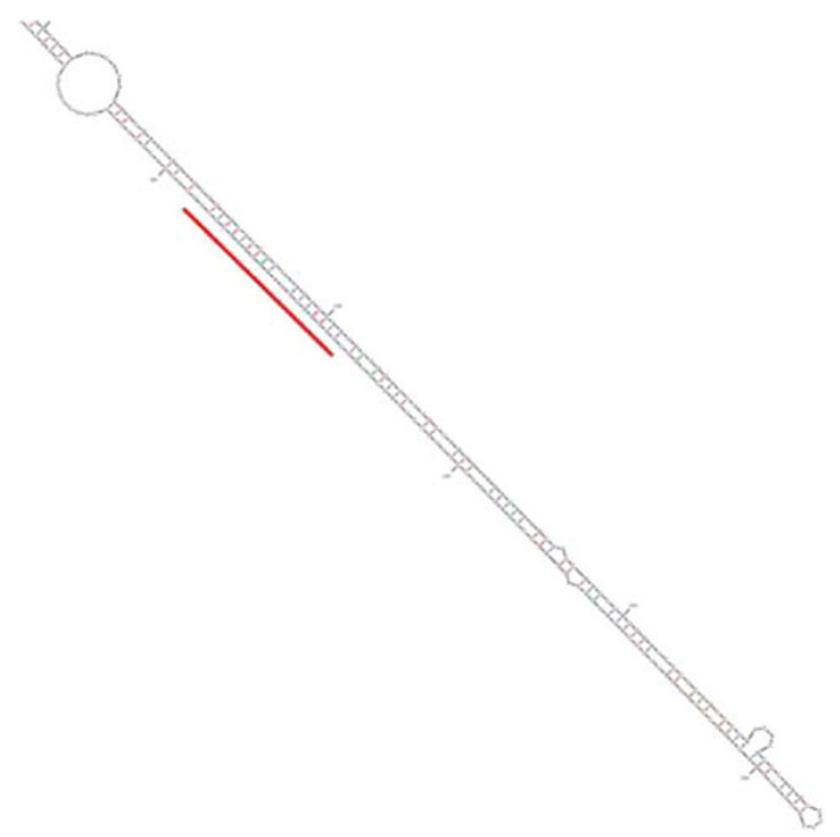

Fig. 5 The predicted secondary structure of candidate miR159 precursor from Diospyros kaki. The secondary structure for EST DC584412.1 (candidate miR159) predicted using MFOLD (Zuker 2003). The sequence encoding the predicted mature miR159 is indicated by the line below the strand in which the mature miRNA is located

hydrolase were observed as major functional domains. Therefore, this strategy not only implicates the evaluation of SSR polymorphisms, but also predicts function viability of these marker sequences. Association between candidate functional markers and trait of interest can be investigated by mapping SSR-FDMs.

miRNA and miRNA target identification

After removal of redundant EST sequences, a total of six ESTs from the MF library and seven from the OYF library were found to align with a known mature miRNA from the plant reference set with fewer than 4 mismatches within the mature miRNA sequence (Table 4). Of these candidates, one EST fulfilled the criteria for miRNA precursors based on the MFE $(-137.40 \mathrm{kcal} / \mathrm{mol})$ and secondary structure as predicted by MFOLD RNA folding program (Zuker 2003). A potential ortholog of miR159 was identified from OYF library (Fig. 5). A discovery rate of one miRNA precursor from a set of 9,457 ESTs of $D$. kaki lies within the expected range as per previous reports, which ranges from 0.83 per 10000 EST reported for Malus domestica to 1.69 per 100,00 EST from Gossypium hirsutum (Qiu et al. 2007; Gleave et al. 2008).

Possible targets of the potential D. kaki miR159 (EST DC584412) were identified using RNA hybrid (Table 4). The candidates were screened and those having an MFE of $-29.7 \mathrm{kcal} / \mathrm{mol}$ or lower (i.e. a minimum of $70 \%$ of the MFE for a perfect match) and two or fewer nucleotide overhangs at either end of the duplex were selected. A perfect match for the mature miRNA159 sequence has a predicted MFE value of $-42.4 \mathrm{kcal} / \mathrm{mol}$ using RNA hybrid. Whilst six ESTs were identified as potential targets of predicted D. kaki miR159, only one of these (EST DC590670) matched an identified protein glutathione S-transferase (Table 5).

Previous reports show that miR159 targets include MYB transcription factors (Jones-Rhoades et al. 2006; Mallory and Vaucheret 2006), however, more recent studies suggest that, along with other miRNA families that are highly conserved across plant species, targets of miR159 are involved in diverse biological processes including gametogenesis, anther development, gibberellins signaling, and ethylene biosynthesis (Alves et al. 2009). Thus, the six EST identified as possible targets in this study may also represent a similar range of diversity.

$\mathrm{GC}_{3}$ biology

Unimodal $\mathrm{GC}_{3}$ profile of $D$. kaki CDS is typical for the Ericales order and other dicot plants (Fig. 6) (Tatarinova 
Table 5 Predicted miRNA159 targets and functions

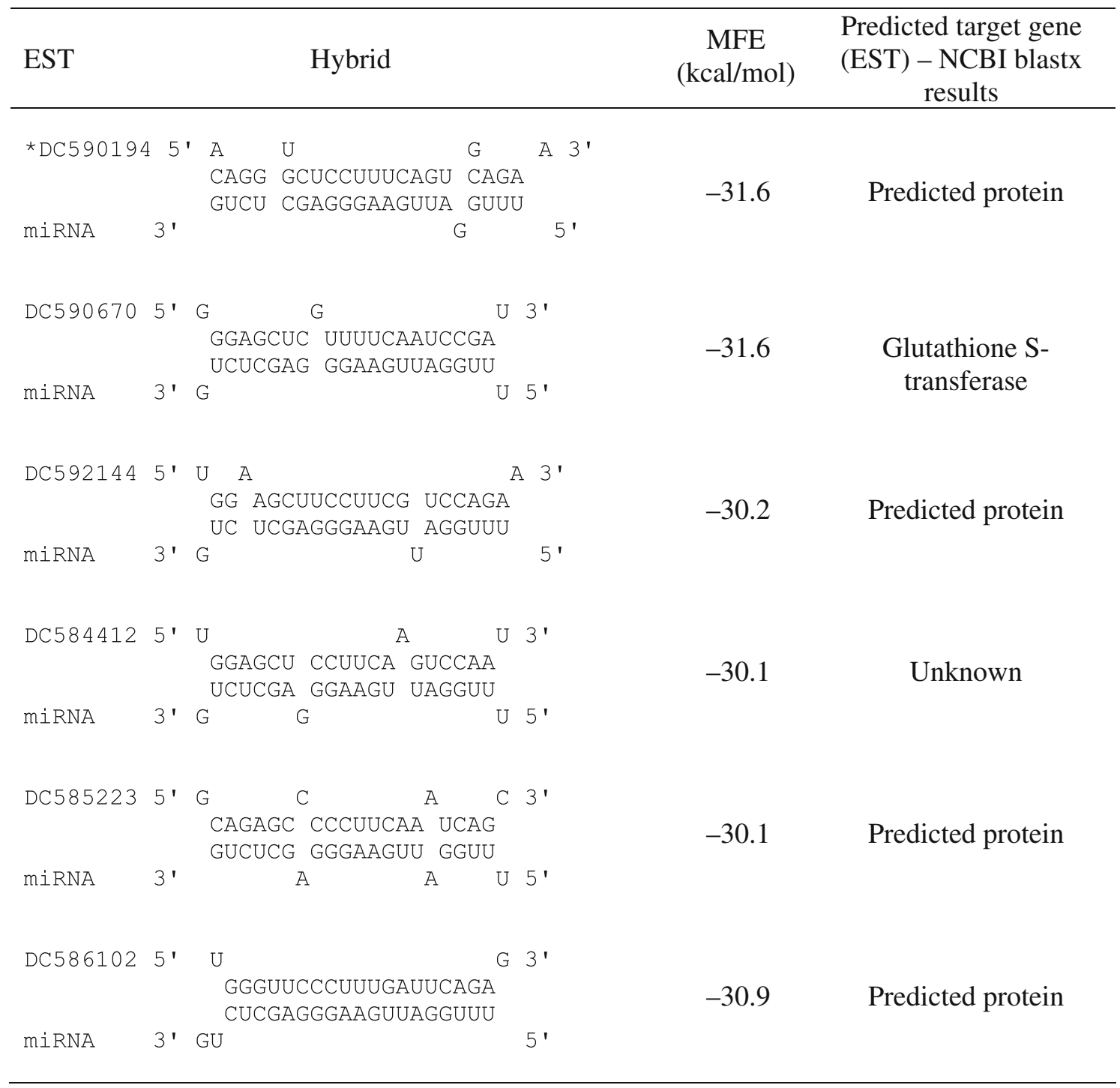

* ESTs DC584412, DC585223 and DC586102 OYF library, whilst DC590194, DC590670 and DC592144 were from the MF library

Fig. 6 GC3 distribution for selected representatives of the Ericales order

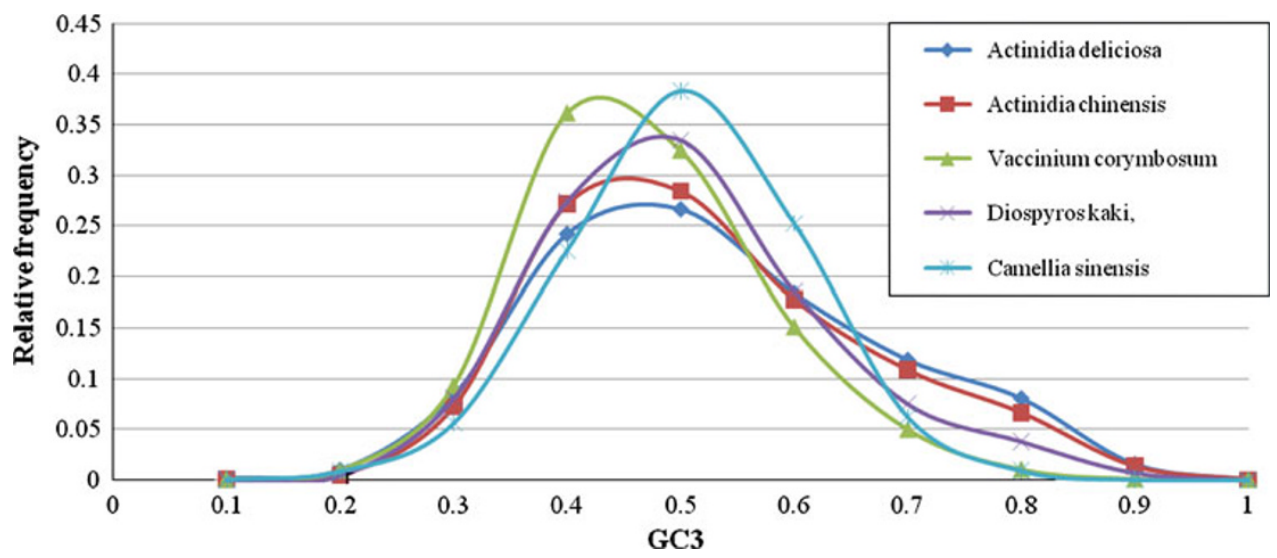


Table 6 GC variations across the different libraries of Diospyros kaki

\begin{tabular}{lllllll}
\hline Library type & Length & $\mathrm{GC}_{1}$ & $\mathrm{GC}_{2}$ & $\mathrm{GC}_{3}$ & $\mathrm{GC}$ & $\mathrm{N}$ \\
\hline Ovary and young fruit library & 177 & 0.51 & 0.45 & 0.50 & 0.49 & 2,322 \\
Mature fruit library & 280 & 0.52 & 0.42 & 0.50 & 0.48 & 2,228 \\
\hline
\end{tabular}

et al. 2010). Both ESTs libraries (OYF and MF) have similar GC content (Table 6). However, the contigs assembled for the mature fruit ESTs library are, on average, approximately
100 nucleotides longer. In order to analyze dependence between $\mathrm{GC}_{3}$ and $\mathrm{GO}$, we took $10 \%$ of highest and lowest genes by $\mathrm{GC}_{3}$ in $D . k a k i$ and four other Ericales genomes ( $A$. chinesis, Actinidia delicosa, V. corymbosum and C. sinesis). According to $\mathrm{GO}$ classification, high $\mathrm{GC}_{3}$ genes are overrepresented in stress response genes, kinases, transcription factors and located in apoplast, membranes and cell wall (Table 7). Low $\mathrm{GC}_{3}$ genes are over-represented in genes involved in protein and nucleotide binding and located in nucleus, cytosol, and cytoplasm.

Table 7 GO-term enrichment for high- and low-GC3 Diospyros kaki genes

\begin{tabular}{|c|c|c|c|c|}
\hline Ontology type & Description & High & Low & Ratio \\
\hline Molecular function & Structural constituent of ribosome & 27 & 2 & 13.500 \\
\hline Cellular localization & Ribosome & 21 & 2 & 10.500 \\
\hline Cellular localization & Apoplast & 18 & 2 & 9.000 \\
\hline Molecular function & Translation & 27 & 3 & 9.000 \\
\hline Molecular function & Response to wounding & 17 & 3 & 5.667 \\
\hline Cellular localization & Endomembrane system & 62 & 13 & 4.769 \\
\hline Cellular localization & Cell wall & 39 & 9 & 4.333 \\
\hline Cellular localization & Endoplasmic reticulum & 25 & 8 & 3.125 \\
\hline Molecular function & Response to salt stress & 26 & 9 & 2.889 \\
\hline Cellular localization & Membrane & 73 & 26 & 2.808 \\
\hline Molecular function & Kinase activity & 25 & 9 & 2.778 \\
\hline Molecular function & Transport & 16 & 6 & 2.667 \\
\hline Molecular function & Protein serine/threonine kinase activity & 16 & 6 & 2.667 \\
\hline Molecular function & Transcription factor activity & 30 & 13 & 2.308 \\
\hline Molecular function & Molecular function & 134 & 59 & 2.271 \\
\hline Cellular localization & Integral to membrane & 18 & 8 & 2.250 \\
\hline Molecular function & Protein amino acid phosphorylation & 19 & 9 & 2.111 \\
\hline Biological process & Biological process & 155 & 75 & 2.067 \\
\hline Cellular localization & Nucleolus & 18 & 9 & 2.000 \\
\hline Cellular localization & Plant-type cell wall & 17 & 9 & 1.889 \\
\hline Cellular localization & Cellular component & 138 & 76 & 1.816 \\
\hline Cellular localization & Vacuole & 41 & 23 & 1.783 \\
\hline Molecular function & Defense response & 14 & 8 & 1.750 \\
\hline Molecular function & Protein binding & 41 & 24 & 1.708 \\
\hline Cellular localization & Chloroplast stroma & 17 & 10 & 1.700 \\
\hline Molecular function & ATP binding & 22 & 13 & 1.692 \\
\hline Cellular localization & Mitochondrion & 37 & 22 & 1.682 \\
\hline Cellular localization & Chloroplast & 97 & 58 & 1.672 \\
\hline Molecular function & DNA binding & 25 & 15 & 1.667 \\
\hline Cellular localization & Chloroplast envelope & 16 & 10 & 1.600 \\
\hline Molecular function & Metabolic process & 20 & 13 & 1.538 \\
\hline Cellular localization & Plasma membrane & 90 & 61 & 1.475 \\
\hline Cellular localization & Nucleus & 56 & 38 & 1.474 \\
\hline Cellular localization & Cytoplasm & 19 & 13 & 1.462 \\
\hline Molecular function & Transferase activity, transferring glycosyl groups & 12 & 9 & 1.333 \\
\hline Molecular function & Response to cadmium ion & 16 & 14 & 1.143 \\
\hline
\end{tabular}


Table 7 continued

\begin{tabular}{|c|c|c|c|c|}
\hline Ontology type & Description & High & Low & Ratio \\
\hline Molecular Function & Catalytic activity & 28 & 25 & 1.120 \\
\hline Molecular function & Zinc ion binding & 13 & 13 & 1.000 \\
\hline Cellular localization & Cytosol & 19 & 21 & 0.905 \\
\hline Molecular function & ATP binding & 26 & 29 & 0.897 \\
\hline Molecular Function & Embryonic development ending in seed dormancy & 13 & 15 & 0.867 \\
\hline Molecular function & Nucleotide binding & 13 & 15 & 0.867 \\
\hline Molecular function & Nucleic acid binding & 12 & 18 & 0.667 \\
\hline Molecular function & Protein binding & 13 & 21 & 0.619 \\
\hline Molecular function & Binding & 13 & 23 & 0.565 \\
\hline
\end{tabular}

The present study was aimed to generate resources that can be utilized for the identification and characterization of D. kaki germplam. The markers identified here can be used for subsequent prediction of germplasm diversity, phylogeography and species discrimination among the Diospyros genus.

Acknowledgments We would like to thank anonymous reviewers for helpful comments. Gaurav Sablok thanks Key Lab of Horticultural Plant Biology (MOE), Huazhong Agricultural University. Jennifer Ann Harikrishna and Lee Wan Sin thank the Centre for Research in Biotechnology for Agriculture (CEBAR) and the Genetics and Molecular Biology Division, Institute of Biological Sciences, Faculty of Science, University of Malaya, Kuala Lumpur, Malaysia for providing computational facilities. Tatiana Tatarinova would like to thank the University of Glamorgan's Research Investment Scheme and the Faculty of Advanced Technology for supporting this project. This work was funded by the Special Scientific Research Fund of Agricultural Public Welfare Profession of China (No. 200903044).

Open Access This article is distributed under the terms of the Creative Commons Attribution License which permits any use, distribution, and reproduction in any medium, provided the original author(s) and source are credited.

\section{References}

Akagi T, Ikegami A, Tsujimoto T, Kobayashi S, Sato A, Kono A, Yonemori K (2009) DkMyb4 is a Myb transcription factor involved in proanthocyanidin biosynthesis in persimmon fruit. Plant Physiol 151:2028-2045

Alves L Jr, Niemeier S, Hauenschild A, Rehmsmeier M, Merkle T (2009) Comprehensive prediction of novel microRNA targets in Arabidopsis thaliana. Nucleic Acids Res 37:4010-4021

Barker G, Batley J, O'sullivan H, Edwards KJ, Edwards D (2003) Redundancy based detection of sequence polymorphisms in expressed sequence tag data using autoSNP. Bioinformatics 19:421-422

Bartel DP (2004) MicroRNAs: genomics, biogenesis, mechanism, and function. Cell 116:281-297

Ching A, Caldwell KS, Jung M, Dolan M, Smith OS, Tingey S, Morgante M, Rafalski AJ (2002) SNP frequency, haplotype structure and linkage disequilibrium in elite maize inbred lines. BMC Genetics 3:19
Da Silva FG, Iandolino A, Al-Kayal F, Bohlmann MC, Cushman MA, Lim H, Ergul A, Figueroa R, Kabuloglu EK, Osborne C, Rowe J, Tattersall E, Leslie A, Xu J, Baek J, Cramer GR, Cushman JC, Cook DR (2005) Characterizing the grape transcriptome. Analysis of expressed sequence tags from multiple Vitis species and development of a compendium of gene expression during berry development. Plant Physiol 139:574-597

Dixon RA (2005) Engineering of plant natural product pathways. Curr Opin Plant Biol 8:329-336

Du XY, Zhang QL, Luo Z (2009a) Comparison of four molecular markers for genetic analysis in Diospyros L. (Ebenaceae). Plant Syst Evol 281:171-181

Du X, Zhang Q, Luo Z (2009b) Development of retrotransposon primers and their utilization for germplasm identification in Diospyros spp. (Ebenaceae). Tree Genet Genomes 5:235-245

FAO (2008) FAOSTAT database. http://faostat.fao.org

Fei Z, Tang X, Alba RM, White JA, Ronning CM, Martin GB, Tanksley SD, Giovannoni JJ (2004) Comprehensive EST analysis of tomato and comparative genomics of fruit ripening. Plant J 40:47-59

Fondon JW, Hammock EA, Hannan AJ, King DG (2008) Simple sequence repeats: genetic modulators of brain function and behavior. Trends Neurosci 31:328-334

Gleave AP, Ampomah-Dwamena C, Berthold S, Dejnoprat S, Karunairetnam S, Nain B, Wang YY, Crowhurst RN, MacDiarmid RM (2008) Identification and characterization of primary microRNAs from apple (Malus domestica cv. Royal Gala) expressed sequence tags. Tree Genet Genomes 4:343-358

Guo D, Luo Z (2006) Genetic relationships of some PCNA persimmons (Diospyros kaki Thunb.) from China and Japan revealed by SRAP analysis. Genet Res Crop Evol 53:1597-1603

Hamblin MT, Mitchell SE, White GM, Gallego J, Kukatla R, Wing RA, Paterson AH, Kresovich S (2004) Comparative population genetics of the panicoid grasses: sequence polymorphism, linkage disequilibrium and selection in a diverse sample of Sorghum bicolor. Genetics 167:471-483

Huang X, Madan A (1999) CAP3: a DNA sequence assembly program. Genome Res 9:868-877

Hytena DL, Choia IY, Songa Q, Spechtc JE, Carter TE, Shoemaker RC, Hwanga EY, Matukumallif LK, Cregana PB (2010) A high density integrated genetic linkage map of soybean and the development of a 1536 universal soy linkage panel for quantitative trait locus mapping. Crop Sci 50:960-968

Ikegami A, Kitajima A, Yonemori K (2005a) Inhibition of flavonoid biosynthetic gene expression coincides with loss of astringency in pollination-constant, non-astringent (PCNA)-type persimmon fruit. J Hortic Sci Biotechnol 80:225-228

Ikegami A, Yonemori K, Kitajima A, Sato A, Yamada M (2005b) Expression of genes involved in proanthocyanidin biosynthesis 
during fruit development in a Chinese pollination-constant, nonastringent (PCNA) persimmon, 'Luo Tian Tian Shi'. J Am Soc Hortic Sci 130:830-835

Ikegami A, Eguchi S, Kitajima A, Inoue K, Yonemori K (2007) Identificaiton of genes involved in proanthocyanidin biosynthesis of persimmon (Diospyros kaki) fruit. Plant Sci 172:10371047

Ikegami A, Akagi T, Potter D, Yamada M, Sato A, Yonemori K, Kitajima A, Inoue KN (2009) Molecular identification of 1-Cys peroxiredoxin and anthocyanidin/Xavonol 3-O-galactosyltransferase from proanthocyanidin-rich young fruits of persimmon (Diospyros kaki Thunb.). Planta 230:841-855

Jian X, Zhang L, Li G, Zhang L, Wang X, Cao X, Fang X, Chen F (2010) Identification of novel stress-regulated microRNAs from Oryza sativa $\mathrm{L}$. Genomics 95:47-55

Jones-Rhoades MW, Bartel DP, Bartel B (2006) MicroRNAS and their regulatory roles in plants. Annu Rev Plant Biol 57:19-53

Kanzaki S, Sato A, Yamada M, Yonemori K, Sugiura A (2001) Identification of molecular markers linked to the trait of natural astringency loss Japanese persimmon (Diospyros kaki) fruit. J Am Soc Hortic Sci 126:51-55

Karaoglu H, Lee CMY, Meyer W (2004) Survey of simple sequence repeats in completed fungal genomes. Mol Biol Evol 22:39-49

Katti MV, Ranjekar PK, Gupta VS (2001) Differential distribution of simple sequence repeats in eukaryotic genome sequences. Mol Biol Evol 18:1161-1167

Mallory AC, Vaucheret H (2006) Functions of microRNAs and related small RNAs in plants. Nat Genet 38(Suppl):S31-S36

Morton BR (1995) Neighboring base composition and transversion/ transition bias in a comparison of rice and maize chloroplast noncoding regions. Proc Natl Acad Sci USA 92:9717-9721

Nasaruddin NM, Harikrishna K, Othman RY, Lim SH, Harikrishna JA (2007) Computational prediction of microRNAs from oil palm (Elaeis guineensis Jacq.) expressed sequence tags. Asia Pac J Mol BiolBiotechnol 15:107-113

Newcomb RD, Crowhurst RN, Gleave AP, Rikkerink EHA, Allan AC, Beuning LL, Bowen JH, Gera E, Jamieson KR, Janssen BJ, Laing WA, McArtney A, Nain B, Ross GS, Snowden KC, Souleyre EJF, Walton EF, Yauk YK (2006) Analyses of expressed sequence tags from apple. Plant Physiol 141:147-166

Pang JH, Ma B, Sun HJ, Ortiz GI, Imanishi S, Sugaya S, Gemma H, Ezura H (2007) Identification and characterization of ethylene receptor homologs expressed during fruit development and ripening in persimmon (Diospyros kaki Thumb.). Postharvest Biol Technol 44:195-203

Park S, Sugimoto N, Larson MD, Beaudry R, Van Nocker S (2006) Identification of genes with potential roles in apple fruit development and biochemistry through large-scale statistical analysis of expressed sequence tags. Plant Physiol 141:811-824

Qiu CX, Xie FL, Zhu YY, Guo K, Huang SQ, Nie L, Yang ZM (2007) Computational identification of microRNAs and their targets in Gossypium hirsutum expressed sequence tags. Gene 395:49-61

Quevillon E, Silventoinen V, Pillai S, Harte N, Mulder N, Apweiler R, Lopez R (2005) InterProScan: protein domains identifier. Nucl Acids Res 33:116-120

Rehmsmeier M (2006) Prediction of microRNA targets. Methods Mol Biol 342:87-99

Rehmsmeier M, Steffen P, Höchsmann M, Giegerich R (2004) Fast and effective prediction of microRNA/target duplexes. RNA 10:1507-1517

Shanker A, Bhargava A, Bajpai R, Singh S, Srivastava S, Sharma V (2007) Bioinformatically mined simple sequence repeats in unigene of Citrus sinensis. Sci Hortic 113:353-361

Somers DJ, Kirkpatrick R, Moniwa M, Walsh A (2003) Mining single-nucleotide polymorphisms from hexaploid wheat ESTs. Genome 46:431-437
Strandberg AKK, Salter LA (2004) A comparison of methods for estimating the transition: transversion ratio from DNA sequences. Mol Phylogenet Evol 32:495-503

Sunkar R, Jagadeeswaran G (2008) In silico identification of conserved microRNAs in large number of diverse plant species. BMC Plant Biol 8:37

Suzuki T, Someya S, Hu F, Tanokura M (2005) Comparative study of catechin compositions in five Japanese persimmons (Diospyros kaki). Food Chem 93:149-152

Tatarinova T, Alexandrov N, Bouck J, Feldmann K (2010) $\mathrm{GC}_{3}$ biology in corn, rice, sorghum and other grasses. BMC Genomics 11:308

The Gene Ontology Consortium (2000) Gene ontology: tool for the unification of biology. Nat Genet 25(1):25-29

Varshney RK, Thiel T, Stein N, Langridge P, Graner A (2002) In silico analysis on frequency and distribution of micro satellites in ESTs of some cereal species. Cell Mol Biol Lett 7:537-546

Varshney RK, Sigmund R, Börner A, Korzun V, Stein N, Sorrells ME, Langridge P, Grane A (2005) Interspecific transferability and comparative mapping of barley EST-SSR markers in wheat, rye and rice. Plant Sci 168:195-202

Varshney RK, Beier U, Khlestkina EK, Kota R, Korzun V, Graner A, Borner A (2007) Single nucleotide polymorphisms in rye (Secale cereale L.): discovery, frequency, and applications for genome mapping and diversity studies. Theor Appl Genet 114:11051116

Wang R, Yang Y, Li G (1997) Chinese persimmon germplasm resources. Acta Hortic 436:43-50

Wu J, Yamagata H, Hayashi-Tsugane M, Hijishita S, Fujisawa M, Shibata M, Ito Y, Nakamura M, Sakaguchi M, Yoshihara R, Kobayashi H, Ito K, Karasawa W, Yamamoto M, Saji S, Katagiri S, Kanamori H, Namiki N, Katayose Y, Matsumoto T, Sasaki T (2004) Composition and structure of the centromeric region of rice chromosome 8. Plant Cell 16:967-976

Xie FL, Huang SQ, Guo K, Xiang AL, Zhu YY, Nie L, Yang ZM (2007) Computational identification of novel microRNAs and targets in Brassica napus. FEBS Lett 581:1464-1474

Yamada M, Sato A (2002) Segregation for fruit astringency type in progenies derived from crosses of 'Nishimura-wase' 3 pollination constant non-astringent genotypes in Oriental persimmon (Diospyros kaki Thunb). Sci Hortic 92:107-111

Yamagishi M, Matsumoto S, Nakatsuka A, Itamura H (2005) Identification of persimmon (Diospyros kaki) cultivars and phenetic relationships between Diospyros species by more effective RAPD analysis. Sci Hortic 105:283-290

Yang T, Xue L, An L (2007) Functional diversity of miRNA in plants. Plant Sci 172:423-432

Yonemori K, Sugiura A, Yamada M (2000) Persimmon genetics and breeding. Plant Breed Rev 19:191-225

Yu JK, Paik H, Choi JP, Han JH, Choe JK, Hur CG (2010) Functional domain marker (FDM): an in silico demonstration in Solanaceae using simple sequence repeats (SSRs). Plant Mol Biol Rep 28:352-356

Zhang BH, Pan XP, Wang QL, Cobb GP, Anderson TA (2005) Identification and characterization of new plant microRNAs using EST analysis. Cell Res 15:336-360

Zhang B, Pan X, Cobb GP, Anderson TA (2006) Plant microRNA: a small regulatory molecule with big impact. Dev Biol 289:3-16

Zhongming Z, Boerwinkle E (2002) Neighboring-nucleotide effects on single nucleotide polymorphisms: a study of 2.6 million polymorphisms across the human genome. Genome Res 12:1679-1686

Zuker M (2003) Mfold web server for nucleic acid folding and hybridization prediction. Nucleic Acids Res 31:3406-3415 